\title{
Studies of rational methods of water selection in water intake areas of hydroelectric power plants
}

\author{
Farrukh Shaazizov ${ }^{1, *}$, Abdulla Badalov ${ }^{1}$, Alisher Ergashev ${ }^{1}$ and Diyor Shukurov ${ }^{2}$ \\ ${ }^{1}$ Tashkent institute of irrigation and agricultural mechanization engineers, Tashkent, Uzbekistan \\ ${ }^{2}$ Saint Petersburg state electro technical university, Saint Petersburg, Russia
}

\begin{abstract}
Up to 2021, 42 new hydropower stations will be created in Uzbekistan and 32 existing hydropower stations will be repaired. Hydraulic engineering designers face a number of problems with the open flow division nodes in the field, which are part of the hydraulic structures of such hydropower plants. Considering the above, the main objectives of the research were determined: a) development of a refined method for the hydraulic calculation of flow division nodes with a quiet flow regime; b) the development of methods for predicting deformation of the channel in the area of the division node. The studies were carried out using theoretical and experimental studies using the equation for changing the amount of movement, laboratory studies on a hydraulic model, field surveys of existing water intake nodes, as well as analysis of experimental data available in the literature on this issue. Based on the theoretical and experimental studies, recommendations were made for the open flow division nodes. These recommendations are valid for division nodes at a division angle not exceeding $90^{\circ}$, for prismatic channels of rectangular cross section with relatively small bottom slopes. These recommendations are intended for flows with values of $\mathrm{B} / \mathrm{H}=3 \div 7$.
\end{abstract}

\section{Introduction}

Solving the problems of hydropower development is one of the main tasks for the Republic of Uzbekistan. The Government of the state approved the Program for the further development of hydropower industry until 2021.

The main objectives of this program are scheduled:

- Increasing the share of renewable energy sources in the country's energy sector;

- Creation of new environmentally friendly generating capacity;

- Providing technical and technological re-equipment of existing hydropower plants;

- Effective water management;

- Improving the balance of energy resources;

- Ensuring the most complete satisfaction of the needs of enterprises and the public in electric power.

\footnotetext{
* Corresponding author: shosfarruh@ mail.ru
} 
Up to 2021, 42 new hydropower stations will be built in Uzbekistan and 32 existing hydropower stations will be repaired. Currently, Uzbekistan has 32 hydropower plants with a total capacity of less than 2 gigawatts, which produce only about $15 \%$ of all electricity in the country. In the next five years, it is planned to substantially change this proportion through the construction of small and medium hydropower plants and the modernization of existing systems.Along with the construction and reconstruction of large hydropower plants, much attention is paid today to the construction of medium and small hydropower plants, including hydroelectric power stations located on small rivers and irrigation canals of the republic. Hydraulic engineering designers face a number of problems with the open flow division nodes in the field, which are part of the hydraulic structures of such hydropower plants.As is known, significant reserves of hydropower resources are concentrated in the mountainous areas of small rivers, in the foothill areas of irrigation canals. In characteristic areas of natural and artificial watercourses, the use of a derivational method of creating pressure in the construction of small hydropower plants is considered most appropriate.These parts of the watercourses are characterized by high velocities of water flow and significant channel deformations. In the water flow of the watercourses in the period of the flood flow, there is a significant increase in the content of suspended and bottom sediments. The passage of water containing a significant amount of sediment leads to a strong hydro abrasive wear of the main hydraulic units and the water-supplying path of the hydroelectric station. With a strong hydro abrasive wear of hydraulic units, there is a sharp decrease in the energy performance of hydraulic units. In addition, hydro abrasive wear of the main hydraulic units and the water-supplying path of a hydroelectric station can contribute to the formation of emergency situations at hydroelectric power stations and can even lead to human victims. To prevent such situations and develop measures to prevent them at the site of water intake from the water source, you must have an idea about the movement of the bottom layer of sediment and the surface layer of suspended sediment. Given the particular importance of this issue, in conducting research, special attention was paid to the study of methods and methods for rational selection of water in the open flow division nodes, which are often found in water intake areas from small rivers and irrigation canals to various hydropower stations.

\section{Goals and objectives of research}

Considering the above, the main objectives of the research were defined, which were as follows:

a) development of a refined method for the hydraulic calculation of flow division nodes with a smooth flow regime;

b) the development of methods for predicting the deformation of the channel in the area of the division node.

This goal is achieved by solving the following tasks:

- the study of the nature of the occurring channel deformations in the area of the division node, as well as the study of the nature of the development and changes in the whirlpool zone in the diversion channel;

- the study of the nature of changes in the bands of the bottom and surface water intakes;

- drawing up recommendations for the reduction of channel deformations.

\section{Methods}

Theoretical and experimental studies using the equation for changing the amount of movement, laboratory studies on the hydraulic model, field surveys of existing water intake 
nodes, as well as analysis of experimental data available in the literature on this issue were used.

\section{Results}

Experimental studies of the separation of flows in rectangular channels carried out on a special hydraulic model installation showed that the overall picture of the division is similar to that noted in the works of many authors [1-20]. Namely, at the beginning of the left bank of the diverting channel, at the entrance edge, a whirlpool zone is formed, the transverse dimensions of which substantially depend on the size of the intake flow. In the alignment, where the whirlpool has the greatest width, the average depth of the flow is the smallest, thereby determining the location of the so-called compressed section. Below the compressed section, there is a gradual decrease in the whirlpool and an increase in the depth of the outflow to the value of the depth corresponding to the domestic flow regime in the channel.

Channel deformations and the nature of changes in the whirlpool zone in the division node Experimental studies have shown that when dividing the flow at the beginning of the diverting channel (on the left bank), a whirlpool region is formed, where there is a decrease in velocity. And the area opposite to this coast is characterized by the formation of a transit flow zone, accompanied by a sharp increase in average speeds. The formation of the whirlpool zone in the diverting channel is caused by a sudden increase in the width of the main channel and rotation of the diverted flow at a certain angle. Due to the latter, under the action of centrifugal forces in the diverting channel, transverse circulation occurs. As a result of transverse circulation, the diverted flow intensively blurs the coast in the transit flow zone, and some of the erosion products are deposited in the whirlpool area and the other is carried away downstream.

Experimental studies of the whirlpool zone were carried out to determine the conditions of formation and the nature of changes in its overall dimensions. However, the task had certain difficulties, since the boundary separating the whirlpool from the transit flow does not have a clear outline. In terms of it is undulating. Moreover, the period and length of such waves are chaotic. A micro-rotating screw installed at some approximate point of the boundary may remain stationary for some time, and then suddenly begin to rotate in the forward or reverse direction and suddenly stop again. The wave-like shape of the boundary of the whirlpool zone appears brighter with a decrease in the flow velocity. In this regard, to obtain numerical values characterizing the width of the zone, the following technique was used.

When conducting experimental studies, a nylon thread approximately 30-40 mm long was attached to the flow, attached to a vertical thin rod, which was slowly moved within the boundary of the whirlpool. The thread at the same time pulled along the channel, thus characterizing the location of the streamlines. The vertical from which the thread began to confirm the presence of streamlined in terms of flow lines and determined the boundary of the width of the whirlpool. It is likely that with a similar method for determining the width of the whirlpool, the absolute error reached $\pm 1 \mathrm{~cm}$.

Experimental studies have shown that determining the final length of the whirlpool zone is very difficult, because the fluid within the whirlpool zone is constantly exchanging with the transit flow fluid and participates in the overall forward movement of the outflow stream. In the area of growth of the whirlpool width, i.e. from the input edge of the water intake to the compressed section, the exchange of the whirlpool fluid and the transit flow is subtle. Here, at the outlet channel wall, in a number of cross sections, reverse flow movement is observed and suspended solid particles, for example, plastic or aluminum powders, lowered 
into the flow, may not (within a few seconds) leave the zone limits, describing chaotic trajectories in it.

At the same time, at the very bottom of the channel, in the same area, there is a longitudinal movement of the fluid with velocities reaching $0.65-0.85$ of the magnitude of the surface velocities of the outflow. As the compressed section is approached, the large bottom longitudinal velocities at the section of the whirlpool zone attenuate and at the same time a longitudinal movement is observed along its entire width and depth, its speed is approximately 3 - 3.5 times less than the average. A feature of this site is that, in addition to the longitudinal velocities, significant transverse components of the velocities arise here. When moving downstream along the whirlpool zone, the values of the transverse components quickly disappear and create the appearance of a sudden termination of the zone across its entire width. However, a careful study reveals on the surface the presence of small vortex funnels, which characterize the presence of a whirlpool in this area.

The relatively active exchange of masses of liquids from the whirlpool and transit flow makes it difficult to determine the final length of the whirlpool based on measurements of longitudinal velocities or by identifying the locations of the current lines using silk or nylon threads. However, a comparison of the results of the measurement of longitudinal velocities and depths, as well as the determination of the location of the streamlines, made it possible to draw the following conclusions.

For a particular water intake design, the width of the whirlpool depends on the size of the water flow to be taken. In the event of an increase in discharge flow, the width of the whirlpool zone decreases. In addition, the location of the compressed section does not correspond to any of the outlets of the discharge channel and is located not across the channel, but along a certain oblique line. The location of the compressed section for a particular construction of the division site is clearly visible when observing the bottom streamlines at the whirlpool. Bottom streamlines were obtained by lowering kapron threads into the flow.

The length of the section of spreading of the separated flow (after a compressed section) considerably exceeds the length of the section from the beginning of the whirlpool zone to the compressed section. Moreover, according to experimental data, this length depends on the flow rate. The bottom line of the current, the envelope of the whirlpool zone and the intersecting vertical 3, and in some cases vertical 2, were taken as the boundary line bounding the zone from the transit flow. So, based on the analysis of the experimental results obtained, it was found that with increasing flow the flow lines more steeply bend around the whirlpool, and with decreasing flow, the flow lines that go around the whirlpool are elongated along the channel, indicating a large length of the flow spreading area.

Processing the experimental data on the character of the change in the dimensions of the whirlpool zone showed that the dependence proposed by Oficerov A.S. can be used to determine the width of the whirlpool zone. [8], particularly,

$$
\begin{array}{ll}
B_{B . \Im}=B_{B}\left(1-\frac{V_{B}}{V_{1}}\right) & \text { when } \frac{V_{B}}{V_{1}}<1 \\
B_{B . \Im}=-B_{B}\left(1-\frac{V_{B}}{V_{1}}\right) & \text { when } \frac{V_{B}}{V_{1}}>1
\end{array}
$$

where: Вв.3.- width of the whirlpool.

As for the length of the whirlpool, as shown by experimental studies, the length of the whirlpool that occurs in the diverting channel depends on the width of the outlet and the 
angle of division. According to the results of experimental studies, a relationship was obtained to determine the length of the whirlpool:

$$
l_{\text {B.3.. }}=N B_{\text {B.3. }}
$$

where the coefficient $\mathrm{N}$ is determined by table 1

Table 1. The value of the coefficient $\mathrm{N}$ at different angles of division

\begin{tabular}{|c|c|c|c|}
\hline angles of division, $\varphi$ & $\mathbf{3 0}^{\mathbf{0}}$ & $\mathbf{6 0}^{\mathbf{}}$ & $\mathbf{9 0}^{\mathbf{0}}$ \\
\hline coefficient $\mathbf{N}$ & 6.36 & 6.81 & 7.75 \\
\hline
\end{tabular}

A comparison of this dependence with the data of our experiments shows that there is good convergence between them and it can be used for practical calculations.

Study of the nature of changes in the bottom and surface water intake bands

One of the most important characteristics of separated flows is the relative width of the surface and bottom water intake bands.

On the basis of the obtained results of the conducted research, the nature of the change in the width of the water intake bands of surface and bottom jets of the water flow was analyzed depending on the change in head flow. As shown by the results of experimental studies, a change in the angle of allotment has practically no effect on the width of the water collection with an increase in the cost ratio Qв / Q1.

Studies show that the relative width of the water intake band for surface $\mathrm{B} \Pi$ / B and bottom Вд / В jets increases with increasing Qв / Q1. On this basis, we carried out detailed studies of the width of the water intake system from measurements of boundary current lines for an angle of $\varphi=30^{\circ}$, depending on the total flow rate of the main flow.

An analysis of the studies conducted to determine the width of the water intake band for surface and bottom jets of a water flow showed that their values can be determined by the following empirical dependencies or graphic dependencies:

$$
\begin{aligned}
& \frac{B_{n}}{B}=0,92\left(\frac{Q_{e}}{Q_{1}}\right)^{1,1} \\
& \frac{B_{\text {K }}}{B}=1,69\left(\frac{Q_{e}}{Q_{1}}\right)^{0,91}
\end{aligned}
$$

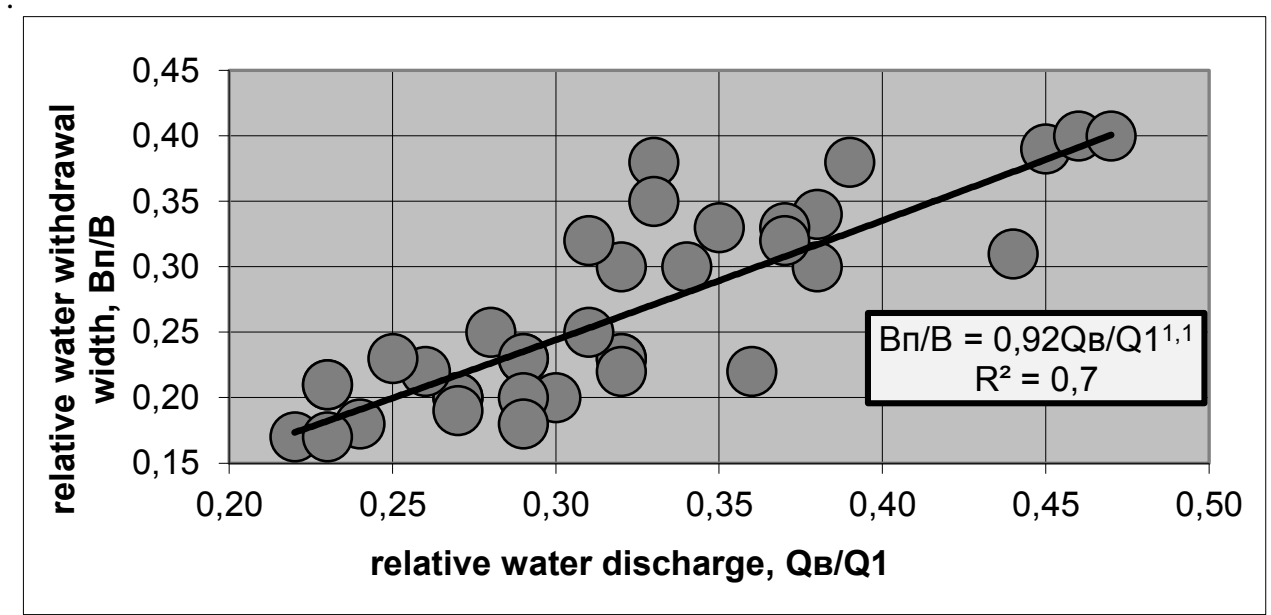

Fig. 1. Graphical dependence of the change in the relative width of the surface jets of the water intake strip. 


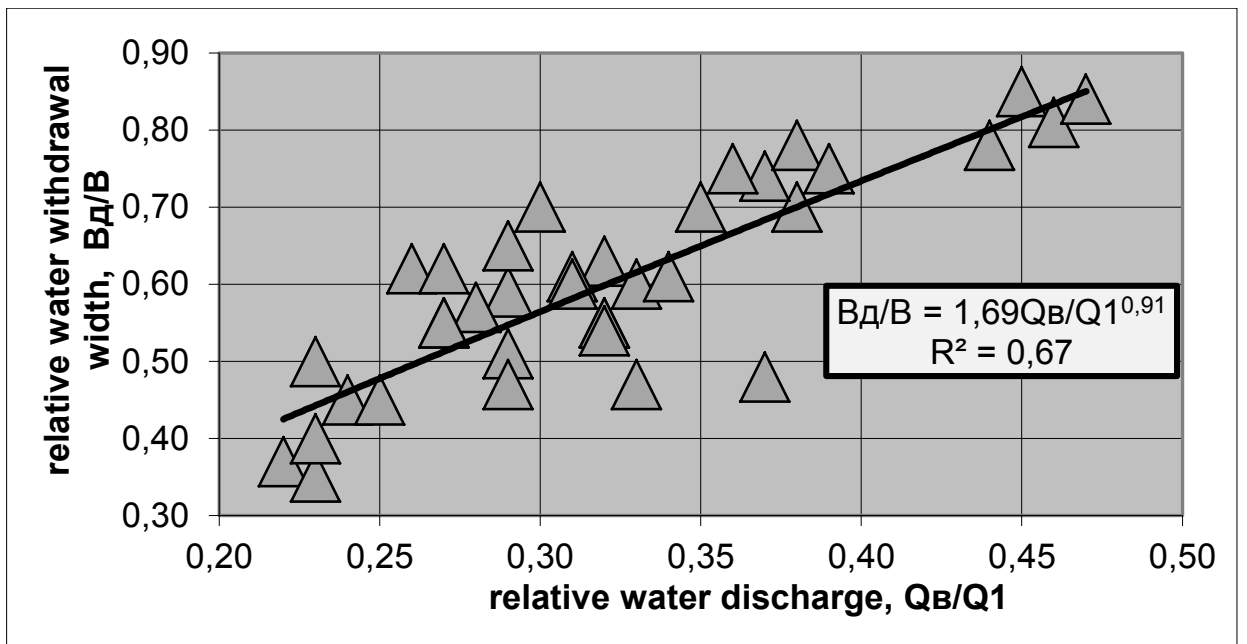

Fig. 2. Graphical dependence of the change in the relative width of the bottom jets of the water intake strip.

\section{Conclusions}

Based on the theoretical and experimental studies, recommendations were made for the open flow division nodes. These recommendations are valid for division nodes at a division angle not exceeding $90^{\circ}$, for prismatic channels of rectangular cross section with relatively small bottom slopes. These recommendations are intended for flows with values $\mathrm{B} / \mathrm{H}=3$ $\div 7$.

Research has shown that when dividing open streams it is noted [1-20]:

- lowering the level of the water surface in the mainstream before the division node;

- education in the diversion channel of the whirlpool;

- occurrence of transverse circulation of the flow;

- redistribution of velocities along the depth and width of the flow.

In the mainstream, the following characteristic zones are noted:

- zone of lowering the water surface. The depths here vary from the household value hlb for the head flow Q1 to the value h1 near the alignment located at the upper edge of the outlet.

- the zone of direct dividing the flow, where there is an increase in depth and decrease in velocity. This zone is located between the upper and lower edges of the outlet.

- flow stabilization zone, where the depth of the main flow goes into the domestic depth $\mathrm{h} 2$ for the remaining flow rate $\mathrm{Q} 2$.

In the diversion channel, the following zones occur:

- education area of the whirlpool area. In this zone, the depth of the diverting channel first decreases from the initial section to the section of the compressed section, and then increases. As for speeds, on the contrary, speeds first increase to a compressed section, and then decrease further.

- flow stabilization zone. It is located below the station of the disappearance of the whirlpool area, where the depths are set to the values of household hв for the discharge of the diverting channel Qв.

Analysis of the studies on the division of quiet open flows showed that significant channel deformations occur at the beginning of the section of the diverting channel, in the zone of formation of the whirlpool area and in the initial section of the main channel. 
To reduce the size of the whirlpool in the diverting channel and the volume of channel deformations, it is necessary to make the division node at a small angle and to adjoin the upper and lower edges of the diverting channel with the main one to produce smoothly by rounding them.

To reduce the channel deformations for the fission sites located on the eroded soils, it is necessary to mount the main channel, starting with the alignment located at a distance of 2B above the upper edge of the outlet to the distance B below the lower edge of the outlet. For the diversion channel, to fix the channel, starting from the initial section of the outlet at a distance of $5 \mathrm{~B}$ in the downstream.

\section{References}

1. F.Sh. Shaazizov Some aspects of the study of rational methods of water selection, Coll. of art. Modern problems of agriculture: The Present and Perspective. Moldova, pp. 6468. (2013)

2. F.Sh. Shaazizov, A.A. Ergashev To the question of the study of channel processes at the site of a dam-free intake, Coll. of art. Improving the efficiency, reliability and safety of hydraulic structures, Tashkent, I , pp. 509-514. (2018)

3. F.Sh. Shaazizov, A.B. Nasrulin Experience in researching topical issues of rational and efficient use of water resources in the lower reaches of the river Amu Darya Inf.and Analit. J. "Ecology and Industry of Kazakhstan" 246 Republic of Kazakhstan, Almaty pp. 48-51. (2015)

4. F.Sh. Shaazizov Research of the non-dammed water intake site at the KMK from the Amudarya River, Coll. of art. Water for Sustainable Development of Central Asia "Water for Sustainable Development, 2018-2028" Dushanbe: PROMEXPO Publishing House, pp. 353 - 358. (2018)

5. F.Sh. Shaazizov To the calculation of the depths in the separation of the stream Coll. of art. IWP AScRUz, 3 Tashkent, pp. 170-176. (1995)

6. F.Sh. Shaazizov Recommendations for hydraulic calculation of open flow division nodes, Coll. of art. Modern problems of hydropower, Tashkent, pp. 38-39. (1997)

7. F.Sh. Shaazizov Hydraulic studies of the open flow division node, Coll. of art. Land Reclamation and Water Management, Tashkent, pp. 103-105. (1997)

8. A.S. Oficerov Questions hydraulics water intake State publishing house of literature on construction and architecture p.235 (1952)

9. A.S. Bryzgalov Influence of the width and angle of the front of a bottomless water intake on the seizure of sediment and floating bodies into the outlet, Reports of the TAA, l (56) pp. 226-231. (1960)

10. N.A. Kartvelishvili Unsteady modes in power units of hydroelectric power stations, Gosenergoizdat p.136. (1951)

11. V.M. Makkaveev Distribution of longitudinal and transverse velocities in open streams GGI 2 (56) pp.126-128. (1947)

12. N.E. Markova The main differential equation for the motion of a fluid with a variable flow rate in two-way dividing flow Hydraulic calculations of canals and structures pp.180-187. (1987)

13. A.S. Marchenko, I.E. Frolova On the question of the influence of the knot of division at the branching of the natural channel, Coll. of art. Hydraulic research facilities. Cleaning liquid. Saratov, pp.67-78. (1982)

14. I.K. Nikitin Turbulent bed flow and processes in the bottom region, Coll. of art. Academy of Sciences, Kiev p.142. (1963)

15. G.A. Petrov Fluid flow with flow variation along the path. Stroiizdat p. 200. (1951)

16. G.A. Petrov Variable mass hydraulics. Kharkov, p.204. (1964) 
17. V.V. Balanin, G.S. Musin Studying the kinematics of the flow in the area of the lateral water intake from channals. Bull. Assoc. inf. permon. Cour. navig. 40 pp.107-117. (1981)

18. H. Bulle Untersuchungen uber die Geschiebeableitung bei der spaltung von Wasserlaufen, Bauwerke, p.98. (1926)

19. F.H. Clauser J. Adv. in Appl. Mec.1(4), New York pp.97- 101. (1956)

20.S. Corrsin, A.L. Kistler The free-stream boundaries of turbulent flows Tech. Notes NACA, Washington, pp. 31-33. (1954) 